\title{
Targeted inhibition of endoplasmic reticulum stress: New hope for renal fibrosis (Review)
}

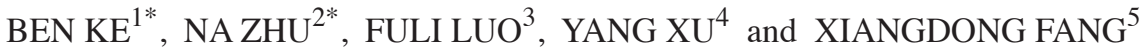 \\ ${ }^{1}$ Department of Nephrology, The Third Hospital of Nanchang, Nanchang, Jiangxi 330009; ${ }^{2}$ Nanchang \\ University School of Medicine; ${ }^{3}$ Department of Nephrology, Chinese Medicine Hospital in Jiangxi \\ Province; ${ }^{4}$ Department of Nephrology, Jiangxi Provincial People's Hospital; ${ }^{5}$ Department of Nephrology, \\ The Second Affiliated Hospital of Nanchang University, Nanchang, Jiangxi 330006, P.R. China
}

Received June 17, 2016; Accepted April 12, 2017

DOI: $10.3892 / \mathrm{mmr} .2017 .6762$

\begin{abstract}
Chronic kidney disease (CKD) has a very high mortality rate and remains a global health challenge. Inhibiting renal fibrosis is one of the most promising therapeutic strategies for CKD. Recent studies have indicated that endoplasmic reticulum stress (ERS) serves an active role in the development of acute and chronic kidney disease, especially with regards to renal fibrosis. In the current review, the authors summarize the latest understanding of the role of ERS during the onset of renal fibrosis. ERS promotes renal fibrosis through multiple signaling pathways, such as transforming growth factor- $\beta$, epithelial-mesenchymal transition and oxidative stress. In addition, ERS also causes podocyte damage, leading to increased proteinuria and the development of renal fibrosis in rat models. In conclusion, targeted inhibition of ERS may become a promising therapeutic strategy for renal fibrosis.
\end{abstract}

\section{Contents}

1. Introduction

2. The cause and role of ERS

3. Relationship between TGF- $\beta$ and ERS

4. Relationship between ERS and EMT

5. Relationship between ERS and oxidative stress

6. Relationship between ERS and proteinuria

7. Potential value of ERS for the treatment of renal fibrosis

8. Conclusions

Correspondence to: Professor Xiangdong Fang, Department of Nephrology, The Second Affiliated Hospital of Nanchang University, 1 Minde Road, Nanchang, Jiangxi 330006, P.R. China E-mail: xiangdongfang818@sina.com

*Contributed equally

Key words: renal fibrosis, Endoplasmic reticulum stress, transforming growth factor- $\beta$, epithelial-mesenchymal transition, oxidative stress, proteinuria

\section{Introduction}

Chronic kidney failure, the endpoint of chronic kidney disease (CKD), is defined as a glomerular filtration rate persistently below $15 \mathrm{ml} / \mathrm{min}$ per $1.73 \mathrm{~m}^{2}$. Renal replacement therapy (RRT) is achieved by hemodialysis, hemodiafiltration, peritoneal dialysis or kidney transplantation, and can be lifesaving for patients with CKD. However, the mortality rate for patients on RRT remains high, and $<25 \%$ of patients with chronic kidney failure undergo RRT in developing countries (1).

Renal fibrosis is a complicated process characterized by fibroblast proliferation and the accumulation of extracellular matrix (ECM). Collectively, these changes lead to renal tubule fibrosis, glomerular sclerosis, renal artery stenosis and chronic inflammatory cell infiltration (2). It is currently believed that renal fibrosis develops in response to ECM accumulation due to epithelial-mesenchymal transition (EMT) (3), transforming growth factor (TGF) $-\beta$ signaling (4), oxidative stress (5) and proteinuria (6). Endoplasmic reticulum stress (ERS) is a physiological or pathological condition that can be caused by glucose deprivation, hypoxia or virus infection. Recent studies have shown that ERS plays a vital role in the development of CKD $(7,8)$. Furthermore, inhibition of ERS can alleviate renal fibrosis progression (9-11). Although a rich body of knowledge regarding the relationship between ERS and CKD has been accumulated to date, how ERS contributes to renal fibrosis has not been fully elucidated. In the present review, the authors summarize the latest understanding of the role of ERS during the onset of renal fibrosis, and propose that targeted inhibition of ERS may become a promising therapeutic strategy for renal fibrosis.

\section{The cause and role of ERS}

The ER performs a variety of cellular function, including the regulation of protein biosynthesis, folding trafficking and modification (8). The ER is implicated in several cellular processes via three major types of signaling: 1) Protein kinase RNA-like endoplasmic reticulum kinase (PERK) signaling, 2) inositol-requiring enzyme 1 (IRE1) signaling, and 3) activating transcription factor (ATF) 6 signaling (12). The PERK pathway is activated by PERK autophosphorylation, 
PERK then phosphorylates eukaryotic initiation factor- $2 \alpha$ (eIF $2 \alpha$ ). Phosphorylated eIF2 $\alpha$ promotes the expression of apoptotic proteins such as CCAAT-enhancer-binding protein homologous protein (CHOP). Similarly, the IRE1 pathway is activated by autophosphorylation of IRE1, which subsequently induces unconventional splicing of X-box-binding protein-1 (XBP1) mRNA (13). In contrast, ATF6 signaling is initiated by ATF6 cleavage by Site 1 protease and Site 2 protease in the Golgi apparatus, and the cleaved subunit (p50ATF6) functions as a transcription factor (14). ER function can change in response to environmental stimuli, such as ischemia, glucose deprivation, or oxidative stress, as well as to genetic mutations, which can result in abnormal protein folding (15). Accumulation of misfolded proteins in the ER lumen induces a range of ER dysfunctions, collectively referred to as ERS (16). To ensure the fidelity of protein folding and prevent the accumulation of unfolded or misfolded proteins, cells experiencing ERS invoke a well-conserved adaptive response known as the unfolded protein response (UPR) to ameliorate cell damage and restore homeostasis (17).

Under prolonged or severe ERS, a variety of molecular chaperones accumulate in the ER lumen, such as glucose-regulated protein 78 (GRP78), GRP94 and calreticulin, which prevent the accumulation of unfolded proteins and facilitate protein folding (18). Moreover, if these adaptive responses fail to alleviate ERS, apoptotic pathways are activated to eliminate damaged cells. CHOP is thought to be a critical mediator of ERS-induced apoptosis (19).

ERS has a crucial role in kidney diseases. ERS can cause cellular damage and lead to renal fibrosis in podocytes, renal tubular cells, glomerular endothelial cells (GEnCs) and mesangial cells $(11,20,21)$, whereas inhibition of ERS can ameliorate renal fibrosis progression $(9,10)$. Collectively, these findings suggested that the inhibition of molecular chaperones during UPR or directly blocking ERS may offer new therapeutic strategies for renal fibrosis.

\section{Relationship between TGF- $\beta$ and ERS}

TGF- $\beta$ is the primary cytokine that causes fibrosis. Smad proteins are highly conserved transcription factors that are central to signal transduction pathways that mediate the numerous effects of TGF- $\beta$ superfamily (22). For example, the inhibition of ERS reduced TGF- $\beta$ activity in an angiotensin II reperfusion model, thereby alleviating myocardial hypertrophy and fibrosis (23). TGF- $\beta$ induced GRP78 expression in human and mouse lung fibroblasts, while the inhibition of GRP78 expression significantly reduced the expression of collagen and $\alpha$-smooth muscle actin ( $\alpha$-SMA), two biomarkers of fibrosis (24). These results indicate that ERS serves a pro-fibrogenic role.

Prolonged or severe ERS can cause kidney cells to undergo apoptosis, resulting in renal fibrosis, while the inhibition of ERS can delay fibrosis development $(6,25)$. ERS-associated proapoptotic signals, including B-cell chronic lymphocytic leukemia/lymphoma 2-associated protein (BAX) expression and caspase-12 and c-Jun N-terminal kinase (JNK) phosphorylation, are activated in the unilateral ureteral obstructed (UUO) kidney. Prolonged ERS attenuates the expression of both unspliced and spliced XBP-1. In addition, prolonged
ERS activates IRE1 $\alpha$-JNK phosphorylation and the expression of protein kinase RNA-like endoplasmic reticulum kinase (PERK), eIF2 $\alpha$, ATF-4, CHOP and cleavage activating transcription factor 6 (cATF6)-CHOP, collectively resulting in ERS-induced apoptosis. TGF- $\beta$ markedly increases the expression of ERS-associated proteins, such as GRP78, CHOP, ATF4, spliced XBP1 and various profibrotic factors, such as $\alpha$-SMA and connective tissue growth factor. Many of these proteins serve as apoptotic markers in renal tubular cells (8). TGF- $\beta$ also activates ERS-mediated proapoptotic signaling via the JNK pathway, which serves an important role in renal fibrosis and mesangial cell apoptosis (26). Furthermore, ERS promotes apoptosis in GEnCs by regulating TGF- $\beta$ expression (21), ultimately causing renal fibrosis. Sox4 (SRY-related HMG box 4) is required for TGF- $\beta$-induced apoptosis, and is widely expressed during mouse embryogenesis and functions in the development of many tissues (27). A recent study reported that TGF- $\beta$ can induce Sox 4 expression, causing pro-apoptotic responses in pancreatic cancer cells associated with EMT-linked repression of Kruppel-like factor 5 (28). It remains unclear whether this mechanism of apoptosis is related to ERS-induced renal fibrosis.

ERS may promote kidney fibroblast differentiation and collagen formation via the upregulation of TGF- $\beta(29,30)$. Studies based on a renal fibrosis model induced by asymmetric dimethylarginine (ADMA) demonstrated that ADMA could activate ERS sensor proteins PERK and IRE1 $\alpha$, which then induce CHOP expression and JNK phosphorylation in GenCs and mesangial cells $(21,31)$. These changes resulted in increased TGF- $\beta$ expression, and ADMA promoted ERS-related apoptosis by increasing TGF- $\beta$ expression (Fig. 1) (21). In contrast, TGF- $\beta$ has been shown to improve ischemia/reperfusion (I/R)-induced myocardial contractile dysfunction in $\mathrm{H} 9 \mathrm{c} 2$ cells via the inhibition of ERS-dependent markers of apoptosis (GRP78, CHOP, caspase-12, and JNK) and the modulation of $\mathrm{Bcl} 2 / \mathrm{Bax}$ expression (32). Further studies are needed to reveal the relationship between ERS and TGF- $\beta$ in different contexts.

\section{Relationship between ERS and EMT}

During EMT, renal epithelial cells are transformed into mesenchymal cells. These mesenchymal cells serve to ameliorate tissue damage, causing ECM accumulation and the production of myofibroblasts, which are key effectors in ECM synthesis and deposition (33). EMT is characterized by a reduction in cell-cell contact, splitting of the basement membrane, reduced expression of proteins such as E-cadherin, nephrin, podocin and zonula occludens-1, cytoskeletal reorganization, transition to a spindle-shaped morphology, and increased expression of mesenchymal markers such as $\alpha$-SMA, vimentin, type I collagen and fibronectin (33). Although a large number of studies have indicated that EMT in the kidney may lead to renal fibrosis, the relationship remains controversial (3).

ERS mediates EMT via several pathways. Advanced oxidation protein products (AOPPs) are formed in response to the reactions between plasma albumin and chlorinated oxidants during oxidative stress (34). AOPPs have been reported to induce podocyte apoptosis, mesangial cell perturbation (35), distal renal tubular epithelial cell hypertrophy and EMT (36). Li et al (37) showed that AOPPs can promote the progression 


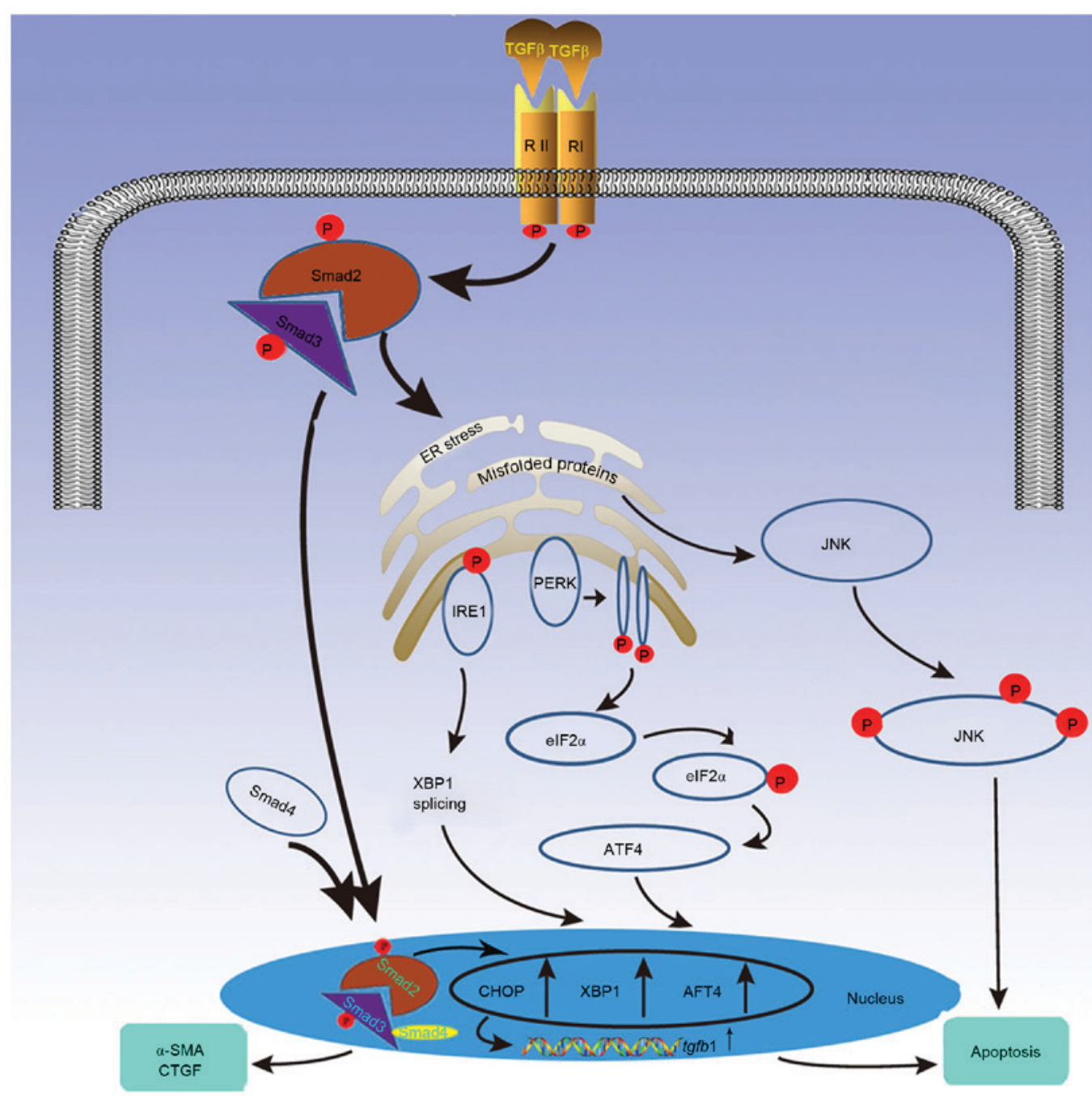

Figure 1. TGF- $\beta$ signaling induced apoptosis is modulated by the UPR cascade. Under severe or prolonged endoplasmic reticulum stress caused by TGF- $\beta$ signaling, UPR stimulates apoptosis through the following 3 proteins: PERK, IRE1 and ATF4. These proteins increase the expression of transcription factors CHOP, XBP1 and ATF4, which promote apoptosis and upregulate TGF- $\beta 1$ expression, thus augmenting TGF- $\beta$ signaling. Additionally, UPR propagates apoptosis via the JNK signaling pathway, which can also be induced by TGF- $\beta$. TGF- $\beta$, transforming growth factor- $\beta$; UPR, unfolded protein response; PERK, protein kinase RNA-like endoplasmic reticulum kinase; IRE1, inositol-requiring enzyme 1; ATF, activating transcription factor; CHOP, CCAAT-enhancer-binding protein homologous protein; XBP1, X-box-binding protein-1; JNK, c-Jun N-terminal kinase; $\alpha$-SMA, $\alpha$-smooth muscle actin.

of early diabetic renal fibrosis. This may be because AOPPs induce ERS by increasing GRP78 and CHOP expression, which causes human renal glomerular endothelial cells to undergo EMT as they age or are exposed to hyperglycemic conditions (35). ERS mediates EMT by decreasing E-cadherin expression and increasing $\alpha$-SMA expression. These AOPP-associated effects can be reversed by treating cells with salubrinal, an inhibitor of ERS, but can be reproduced by treating cells with thapsigargin (TG), an inducer of ERS (35).

In addition, autophagy is induced during stress, and it can alternately contribute to cell death or serve as a cellular survival mechanism (38). ERS resulting from tunicamycin or TG treatment can induce EMT by causing autophagy via c-Src kinase activation in tubular epithelial cells, ultimately leading to renal fibrosis (Fig. 2) (39). Neuronal precursor cell-expressed developmentally downregulated protein (Nedd) 4-2, a $120 \mathrm{kDa}$ highly conserved E3 ligase present in eukaryotic cells, has been demonstrated to regulate membrane availability of a number of ion channels (40). Nedd4-2 has also been reported to be involved in renal-tubular metabolism, leading to kidney injury (41). Wang et al (42) demonstrated that Nedd4-2 is upregulated in response to ER stress by the spliced form of XBP-1, which is important for inducing an appropriate autophagic response in liver cells. Nonetheless, there is still no any report on the relationship between ER stress and Nedd4-2 in kidney cells.

Finally, ERS causes EMT by increasing TGF- $\beta$ expression in renal tubular epithelial cells, and TGF- $\beta$ acts as a major regulator in inducing EMT through the PI3K/Akt pathway, the Notch signaling pathway, the Wnt/ $\beta$-catenin pathway and, perhaps, the AOPPs $(26,43)$. Snail, Zeb1, and TWIST1, as transcriptional factors, have been involved in the process of EMT (44). ERS induces EMT also via increasing the expression of Snail (45), Zeb1 and TWIST1 in cancer cells (44).

\section{Relationship between ERS and oxidative stress}

Oxidative stress is usually produced by reactive oxygen species (ROS). Oxidative stress is common in CKD and serves an important role in renal fibrosis progression (5). ROS attack, 


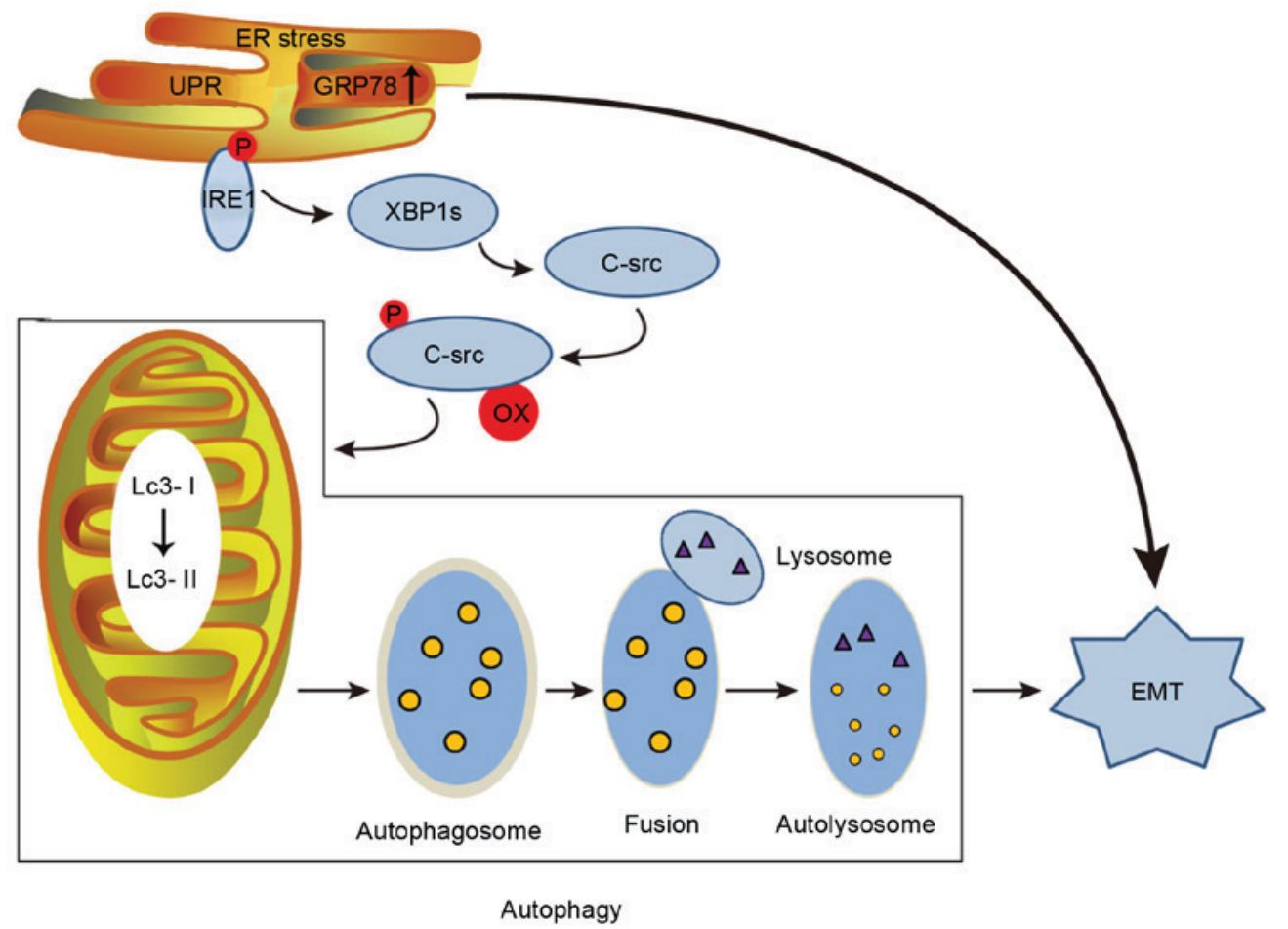

Figure 2. The possible mechanism by which ERS induces EMT. UPR impacts EMT both directly and indirectly. In the former case, GRP78 can increase the expression of $\alpha$-SMA and decrease the expression of E-cadherin, both biomarkers of EMT. In the latter case, ERS stimulates the expression of $\alpha$-SMA through autophagy induced by C-Src. ERS, endoplasmic reticulum stress; EMT, epithelial-mesenchymal transition; UPR, unfolded protein response; GRP78, glucose-regulated protein 78; $\alpha$-SMA, $\alpha$-smooth muscle actin.

denature and modify the structure and function of molecules and activate redox-sensitive transcription factors and signaling pathways, resulting in tissue injury and dysfunction. These events increase ECM expression and promote the development of renal fibrosis (5).

ERS promotes ROS production through multiple pathways. Protein disulfide isomerase (PDI) is an essential enzyme that mediates disulfide bond formation in the ER. In chaperone-assisted disulfide bond formation between peptide chains, two electrons are provided to a cysteine residue within the PDI active site (46). The transfer of electrons reduces the PDI active site and causes substrate oxidation, contributing to ROS production in the ER (47). The NADPH oxidase protein family has seven members, Nox 1-5 and Duox 1 and 2, among them, Nox4 is most commonly associated with the ER (47). ERS and ROS production are fundamental components in the acute and chronic conditions that result from UPR signaling. Following UPR activation, peripheral vasculature cells experience increased Nox4 level, which in turn stimulates ROS production (48). In addition, oxidative stress has also been indicated to initiate and contribute to ERS (49). Therefore, ERS and ROS have a cause-and-effect relationship with each other.

The interaction between ERS and oxidative stress is considered the primary driving force of renal fibrosis. Hyperglycemia commonly leads to oxidative stress. Using a type 2 diabetic rat model, Lee et al (11) reported that ERS and oxidative stress activated TGF- $\beta / \mathrm{Smad} 2 / 3$ signaling and increased $\alpha$-SMA expression, resulting in kidney cell fibrosis and apoptosis and diabetic nephropathy (DN). Recent studies have indicated that aldosterone/mineralocorticoid receptor (MR) is a major contributor to CKD progression (50). For instance, oxidant stress-mediated aldosterone/MR-induced podocyte injury occurs in response to ERS, which then triggers both apoptosis and autophagy to cope with the injury (50).

\section{Relationship between ERS and proteinuria}

CKD is characterized by abnormalities in the glomerular filtration barrier that lead to increased glomerular permeability and abnormal filtration of macromolecules, such as albumin. Evidence from clinical and experimental studies indicates that albuminuria and proteinuria are not simply markers of CKD progression, but rather are active players in the development of CKD (6). Mechanistically, it has been proposed that proteins released into the glomerular filtrate have toxic effects on tubular cells and damaged tubular cells lead to the development of interstitial fibrosis (51).

Podocyte injury is a major cause of proteinuria, and ERS serves an essential role in podocyte damage. ERS was presented to have a close relationship with podocyte injury in a CKD rat model, ERS induces a series of changes such as the upregulation of ER chaperone and ERS proteins, increased podocyte apoptosis, accumulation of mutated and misfolded proteins in the ER, and disruption of the glomerular filtration structure, leading to proteinuria (Fig. 3) (52,53). These findings suggested that UPR augmentation therapy may enhance ER proteostasis and serve as a new therapeutic approach for renal fibrosis.

ERS is common in the pathogenic microenvironment and contributes to the progression of various podocyte diseases. A proteomic study revealed a series of ERS proteins that are mainly involved in cytoskeletal rearrangement, suggesting a potential 


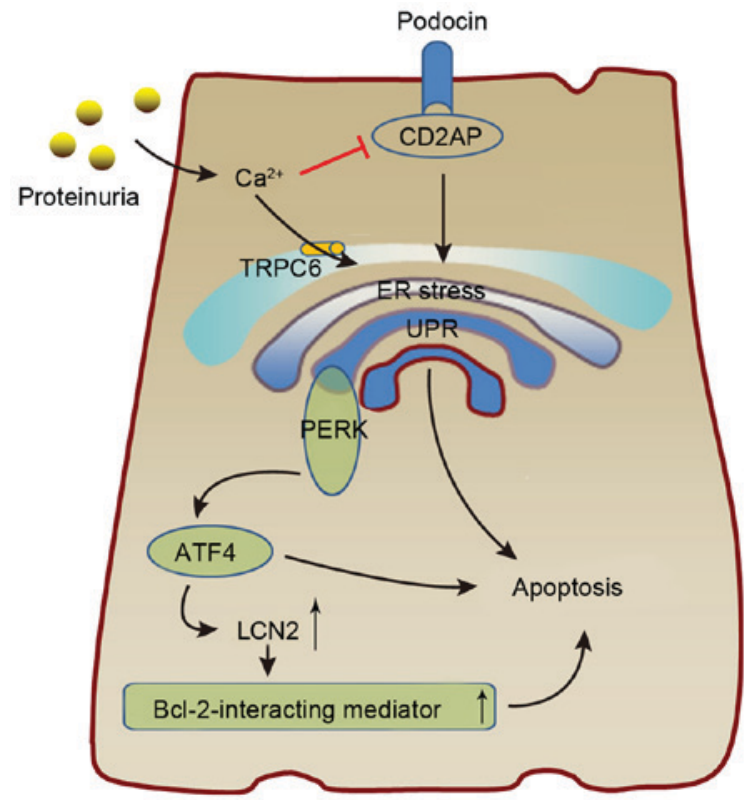

Figure 3. The possible mechanism by which ERS results in podocyte damage. Proteinuria causes ERS via $\mathrm{Ca}^{2+}$, resulting in increased expression of ATF4 and $\mathrm{CHOP}$, which interact to induce LCN2 expression and cause podocyte apoptosis. Additionally, increasing $\mathrm{Ca}^{2+}$ levels can promote podocyte UPR by inhibiting CD2AP, which serves a critical role in podocyte biology. ERS endoplasmic reticulum stress; ATF, activating transcription factor; $\mathrm{CHOP}$, CCAAT-enhancer-binding protein homologous protein; LCN2, lipocalin 2 UPR, unfolded protein response; CD2AP, CD2-associated protein; TRPC6, transient receptor potential C6; PERK, protein kinase RNA-like endoplasmic reticulum kinase.

mechanism by which these proteins cause podocyte dysfunction (54). In addition, abnormal protein accumulation associated with ERS in podocytes produces damage to the cells, which in turn leads to severe proteinuria (55). Slit diaphragm proteins such as CD2-associated protein (CD2AP) serve a critical role in podocyte biology, protein permeability, cell signaling and disease (52). The transient receptor potential (TRP) superfamily member TRPC6 is confirmed to be the primary $\mathrm{Ca}^{2+}$-permeable ion channel in non-excitable cells. TRPC6, a glomerular slit diaphragm-associated channel required for normal renal function, is expressed in the cell body, major processes and foot processes near the slit diaphragm(56). Downregulation of TRPC6 expression ameliorated puromycin aminonucleoside-induced podocyte apoptosis (57). Furthermore, in several studies of albumin overload, which mimics proteinuria, TRPC6-mediated calcium entry into the cells and CD2AP downregulation could induce UPR-mediated apoptosis in podocytes $(52,58)$. ERS also causes podocyte damage through monocyte chemotactic protein-1, which is associated with renal fibrosis-related chronic inflammation (59). Moreover, the activation of rapamycin-sensitive protein kinase complex mTORC1 triggered UPR activation in podocytes in an animal model of $\mathrm{DN}$, leading to podocyte injury (60). The carrier protein neutrophil gelatinase-associated lipocalin (NGAL), also known as lipocalin (LCN) 2, is associated with renal fibrosis and has been demonstrated to promote CKD progression in mice and humans (61). Proteinuria caused by calcium release-induced ER stress results in LCN2 overexpression, which in turn leads to tubular apoptosis and renal lesions. Moreover, 4-phenylbutyric acid (PBA) was reported to delay renal deterioration in proteinuric mice (61). This may be because albumin induces ER stress and increases cytosolic $\mathrm{Ca}^{2+}$ concentrations in cultured podocytes through the activation of TRPC6. In albumin-overloaded tubular cells exhibiting UPR, ERS increases the expression of CHOP and ATF4, and these proteins then interact with other factors to induce LCN2 expression (Fig. 3) (58).

\section{Potential value of ERS for the treatment of renal fibrosis}

Recently, growing evidence has suggested that inhibiting ERS can alleviate the progression of renal fibrosis. Downregulation of Klotho, a transmembrane protein primarily expressed in kidney distal tubular cells, has been indicated to cause multiple age-associated disorders (10). Aging is related to CKD development, and Klotho, which has anti-aging properties, has been implicated in the pathogenesis of various kidney diseases (62). Liu et al (10) reported decreased Klotho expression and the presence of ERS in a UUO model, and Klotho administration was able to ameliorate UUO-induced ER stress, inhibit apoptosis and attenuate renal fibrosis. Another study reported that CHOP deficiency not only attenuates apoptosis and oxidative stress in experimental renal fibrosis, but also reduces local inflammation and ameliorates UUO-induced renal fibrosis (63).

Although some drugs have shown the ability to suppress ERS, further experimental studies are required. The chemical chaperone sodium 4-BPA, an aromatic fatty acid analog, has been used to treat urea cycle disorders because its metabolites offer an alternative pathway to the urea cycle, allowing for excretion of excess nitrogen (8). Liu et al (8) reported that 4-PBA acts as an ER chaperone to ameliorate ERS-induced renal tubular cell apoptosis and renal fibrosis. Valproate (VPA) is a histone deacetylation enzyme inhibitor that increases histone acetylation and promotes gene transcription. VPA has previously been used as an antiepileptic and anti-tumor treatment. Recently, Sun et al (9) demonstrated in a rat model that VPA relieved ERS and reduced renal cell apoptosis, thereby attenuating renal injury. Furthermore, a pentacyclic triterpenoid compound oleanolic acid (OA) has shown therapeutic efficacy for CKD, without apparent side effects. Several studies have reported that OA has anti-oxidant, microbicidal, anti-diabetic, anti-inflammatory, hypolipidemic and anti-atherosclerotic activities (11). In mammals, $\mathrm{N}$-acetylcysteine (NAC, 2-acetamido-3-sulfanylpropanoic acid) is a precursor of intracellular cysteine and glutathione, and a previous study reported that NAC has powerful anti-oxidant and protective effects in $\beta$-cells in diabetic $\mathrm{db} / \mathrm{db}$ mice (64). Lee et al (11) reported that OA and NAC have potential therapeutic effects for DN based on their anti-oxidant effects and ability to reduce ERS. Sodium citrate also has a protective effect on chronic renal failure by affecting ERS (65).

Mesencephalic astrocyte-derived neurotrophic factor (MANF) localizes to the ER lumen and is secreted in response to ERS in several cell types. Using a rat model, Kim et al (66) demonstrated that MANF can potentially serve as an urine diagnostic or prognostic biomarker for ERS-related kidney disease to help stratify disease risk, predict disease progression, monitor treatment response and identify subgroups of patients who can be treated with ER stress modulators in a highly targeted manner. 


\section{Conclusions}

It is important to note that stress is a non-specific systemic protection response and ERS acts as a self-protecting regulatory system to promote cell survival under different stresses that can result in various pathological conditions. However, abnormal or excessive ERS would result in different pathological changes. Therefore, ERS inhibitors not only inhibit fibrosis in the kidney but also alleviate pathological role of excessive ERS in other organs. In summary, targeted inhibition of ERS is of great value for renal fibrosis therapy, and it is expected that ERS inhibitors with clinical applications will become available in the future.

\section{Acknowledgements}

The authors would like to thank Fuli Luo and Na Zhu for their assistance in the creation of the present article. The study was supported by a grant from the National Natural Science Foundation of China (grant no. 81460142).

\section{References}

1. Ortiz A, Covic A, Fliser D, Fouque D, Goldsmith D, Kanbay M, Mallamaci F, Massy ZA, Rossignol P, Vanholder R, et al: Epidemiology, contributors to, and clinical trials of mortality risk in chronic kidney failure. Lancet 383: 1831-1843, 2014.

2. Ke B, Fan C, Yang L and Fang X: Matrix Metalloproteinases-7 and Kidney Fibrosis. Front Physiol 8: 21, 2017.

3. Menon MC and Ross MJ: Epithelial-to-mesenchymal transition of tubular epithelial cells in renal fibrosis: A new twist on an old tale. Kidney Int 89: 263-266, 2016.

4. Meng XM, Huang XR, Xiao J, Chung AC, Qin W, Chen HY and Lan HY: Disruption of Smad4 impairs TGF- $\beta / S m a d 3$ and Smad7 transcriptional regulation during renal inflammation and fibrosis in vivo and in vitro. Kidney Int 81: 266-279, 2012.

5. Habib SL and Abboud HE: Tuberin regulates reactive oxygen species in renal proximal cells, kidney from rodents, and kidney from patients with tuberous sclerosis complex. Cancer Sci 107: 1092-1100, 2016.

6. Xiao W, Fan Y, Wang N, Chuang PY, Lee K and He JC: Knockdown of RTN1A attenuates ER stress and kidney injury in albumin overload-induced nephropathy. Am J Physiol Renal Physiol 310: F409-F415, 2016.

7. Chang JW, Kim H, Baek CH, Lee RB, Yang WS and Lee SK: Up-regulation of SIRT1 reduces endoplasmic reticulum stress and renal fibrosis. Nephron 133: 116-128, 2016.

8. Liu SH, Yang CC, Chan DC, Wu CT, Chen LP, Huang JW, Hung KY and Chiang CK: Chemical chaperon 4-phenylbutyrate protects against the endoplasmic reticulum stress-mediated renal fibrosis in vivo and in vitro. Oncotarget 7: 22116-22127, 2016.

9. Sun XY, Qin HJ, Zhang Z, Xu Y, Yang XC, Zhao DM, Li XN and Sun LK: Valproate attenuates diabetic nephropathy through inhibition of endoplasmic reticulum stress-induced apoptosis. Mol Med Rep 13: 661-668, 2016.

10. Liu QF, Ye JM, Deng ZY, Yu LX, Sun Q and Li SS: Ameliorating effect of Klotho on endoplasmic reticulum stress and renal fibrosis induced by unilateral ureteral obstruction. Iran J Kidney Dis 9: 291-297, 2015.

11. LeeES, Kim HM,Kang JS, Lee EY, Yadav D, Kwon MH, Kim YM, Kim HS and Chung $\mathrm{CH}$ : Oleanolic acid and $\mathrm{N}$-acetylcysteine ameliorate diabetic nephropathy through reduction of oxidative stress and endoplasmic reticulum stress in a type 2 diabetic rat model. Nephrol Dial Transplant 31: 391-400, 2016.

12. Yoshida H, Matsui T, Yamamoto A, Okada T and Mori K: XBP1 mRNA is induced by ATF6 and spliced by IRE1 in response to ER stress to produce a highly active transcription factor. Cell 107: 881-891, 2001

13. Chiang CK, Hsu SP, Wu CT, Huang JW, Cheng HT, Chang YW, Hung KY, Wu KD and Liu SH: Endoplasmic reticulum stress implicated in the development of renal fibrosis. Mol Med 17: 1295-1305, 2011
14. Ye J, Rawson RB, Komuro R, Chen X, Davé UP, Prywes R, Brown MS and Goldstein JL: ER stress induces cleavage of membrane-bound ATF6 by the same proteases that process SREBPs. Mol Cell 6: 1355-1364, 2000.

15. Zeeshan HM, Lee GH, Kim HR and Chae HJ: Endoplasmic reticulum stress and associated ROS. Int J Mol Sci 17: 327, 2016.

16. Kaufman RJ: Stress signaling from the lumen of the endoplasmic reticulum: Coordination of gene transcriptional and translational controls. Genes Dev 13: 1211-1233, 1999.

17. Inagi R: Endoplasmic reticulum stress in the kidney as a novel mediator of kidney injury. Nephron Exp Nephrol 112: E1-E9, 2009.

18. Boot-Handford RP and Briggs MD: The unfolded protein response and its relevance to connective tissue diseases. Cell Tissue Res 339: 197-211, 2010.

19. Ghosh AP, Klocke BJ, Ballestas ME and Roth KA: CHOP potentially co-operates with $\mathrm{FOXO} 3 \mathrm{a}$ in neuronal cells to regulate PUMA and BIM expression in response to ER stress. PLoS One 7: e39586, 2012.

20. Yuan Y, Xu X, Zhao C, Zhao M, Wang H, Zhang B, Wang N, Mao H, Zhang A and Xing C: The roles of oxidative stress, endoplasmic reticulum stress, and autophagy in aldosterone/mineralocorticoid receptor-induced podocyte injury. Lab Invest 95: 1374-1386, 2015.

21. Guo W, Ding J, Zhang A, Dai W, Liu S, Diao Z, Wang L, Han X and Liu W: The inhibitory effect of quercetin on asymmetric dimethylarginine-induced apoptosis is mediated by the endoplasmic reticulum stress pathway in glomerular endothelial cells. Int J Mol Sci 15: 484-503, 2014.

22. Ke B, Zhang A, Wu X and Fang X: The role of Krüppel-like factor 4 in renal fibrosis. Front Physiol 6: 327, 2015.

23. Kassan M, Galán M, Partyka M, Saifudeen Z, Henrion D, Trebak M and Matrougui K: Endoplasmic reticulum stress is involved in cardiac damage and vascular endothelial dysfunction in hypertensive mice. Arterioscler Thromb Vasc Biol 32: 1652-1661, 2012.

24. Roberson EC, Tully JE, Guala AS, Reiss JN, Godburn KE, Pociask DA, Alcorn JF, Riches DW, Dienz O, Janssen-Heininger YM and Anathy V: Influenza induces endoplasmic reticulum stress, caspase-12-dependent apoptosis, and c-Jun N-terminal kinase-mediated transforming growth factor- $\beta$ release in lung epithelial cells. Am J Respir Cell Mol Biol 46: 573-581, 2012.

25. Marek I, Lichtneger T, Cordasic N, Hilgers KF, Volkert G, Fahlbusch F, Rascher W, Hartner A and Menendez-Castro C: Alpha8 integrin (Itga8) signalling attenuates chronic renal interstitial fibrosis by reducing fibroblast activation, not by interfering with regulation of cell turnover. PLoS One 11: e0150471, 2016.

26. Sutariya B, Jhonsa D and Saraf MN: TGF- $\beta$ : The connecting link between nephropathy and fibrosis. Immunopharmacol Immunotoxicol 38: 39-49, 2016.

27. Vervoort SJ, van Boxtel R and Coffer PJ: The role of SRY-related HMG box transcription factor 4 (SOX4) in tumorigenesis and metastasis: Friend or foe? Oncogene 32: 3397-3409, 2013.

28. David CJ, Huang YH, Chen M, Su J, Zou Y, Bardeesy N, Iacobuzio-Donahue CA and Massagué J: TGF- $\beta$ tumor suppression through a lethal EMT. Cell 164: 1015-1030, 2016.

29. Baek HA, Kim DS, Park HS, Jang KY, Kang MJ, Lee DG, Moon WS, Chae HJ and Chung MJ: Involvement of endoplasmic reticulum stress in myofibroblastic differentiation of lung fibroblasts. Am J Respir Cell Mol Biol 46: 731-739, 2012.

30. Guo W, Ding J, Zhang A, Dai W, Liu S, Diao Z, Wang L, Han X and Liu W: The inhibitory effect of quercetin on asymmetric dimethylarginine-induced apoptosis is mediated by the endoplasmic reticulum stress pathway in glomerular endothelial cells. Int J Mol Sci 15: 484-503, 2014.

31. Park MJ, Oh KS, Nho JH, Kim GY and Kim DI: Asymmetric dimethylarginine (ADMA) treatment induces apoptosis in cultured rat mesangial cells via endoplasmic reticulum stress activation. Cell Biol Int 40: 662-670, 2016.

32. Wang Y,Zong L and Wang X: TGF- $\beta$ improves myocardial function and prevents apoptosis induced by anoxia-reoxygenation, through the reduction of endoplasmic reticulum stress. Can J Physiol Pharmacol 94: 9-17, 2016.

33. Son $\mathrm{H}$ and Moon A: Epithelial-mesenchymal transition and cell invasion. Toxicol Res 26: 245-252, 2010.

34. Cao W, Hou FF and Nie J: AOPPs and the progression of kidney disease. Kidney Int Suppl (2011) 4: 102-106, 2014. 
35. Liang X, Duan N, Wang Y, Shu S, Xiang X, Guo T, Yang L, Zhang S, Tang $X$ and Zhang J: Advanced oxidation protein products induce endothelial-to-mesenchymal transition in human renal glomerular endothelial cells through induction of endoplasmic reticulum stress. J Diabetes Complications 30: 573-579, 2016.

36. Tang X, Rong G, Bu Y, Zhang S, Zhang M, Zhang J and Liang X: Advanced oxidation protein products induce hypertrophy and epithelial-to-mesenchymal transition in human proximal tubular cells through induction of endoplasmic reticulum stress. Cell Physiol Biochem 35: 816-828, 2015.

37. Li HY, Hou FF, Zhang X, Chen PY, Liu SX, Feng JX, Liu ZQ, Shan YX, Wang GB, Zhou ZM, et al: Advanced oxidation protein products accelerate renal fibrosis in a remnant kidney model. J Am Soc Nephrol 18: 528-538, 2007.

38. Luo B, Lin Y, Jiang S, Huang L, Yao H, Zhuang Q, Zhao R, Liu H, $\mathrm{He} C$ and Lin Z: Endoplasmic reticulum stress eIF2 $\alpha$-ATF4 pathway-mediated cyclooxygenase- 2 induction regulates cadmium-induced autophagy in kidney. Cell Death Dis 7: e2251, 2016.

39. Moon SY, Kim HS, Nho KW, Jang YJ and Lee SK: Endoplasmic reticulum stress induces epithelial-mesenchymal transition through autophagy via activation of c-Src kinase. Nephron Exp Nephrol 126: 127-140, 2014.

40. Goel P, Manning JA and Kumar S: NEDD4-2 (NEDD4L): The ubiquitin ligase for multiple membrane proteins. Gene 557: 1-10, 2015.

41. Al-Qusairi L, Basquin D, Roy A, Stifanelli M, Rajaram RD, Debonneville A, Nita I, Maillard M, Loffing J, Subramanya AR and Staub O: Renal-tubular SGK1 deficiency causes impaired $\mathrm{K}^{+}$excretion via the loss of regulation of NEDD4-2/WNK1 and ENaC. Am J Physiol Renal Physiol 311: F330-F342, 2016.

42. Wang H, Sun RQ, Camera D, Zeng XY, Jo E, Chan SM, Herbert TP, Molero JC and Ye JM: Endoplasmic reticulum stress up-regulates Nedd4-2 to induce autophagy. FASEB J 30 2549-56, 2016

43. Xian LW, Li TP, Wei YE, Wu SP and Ma L: Relation of advanced oxidation protein products with VEGF and TGF- $\beta 1$ in colon cancer cells exposed to intermittent hypoxia. Nan Fang Yi Ke Da Xue Xue Bao 31: 619-623, 2011 (In Chinese).

44. Granados-Principal S, Liu Y, Guevara ML, Blanco E, Choi DS, Qian W, Patel T, Rodriguez AA, Cusimano J, Weiss HL, et al: Inhibition of iNOS as a novel effective targeted therapy against triple-negative breast cancer. Breast Cancer Res 17: 25, 2015

45. Shin HS, Ryu ES, Oh ES and Kang DH: Endoplasmic reticulum stress as a novel target to ameliorate epithelial-to-mesenchymal transition and apoptosis of human peritoneal mesothelial cells. Lab Invest 95: 1157-1173, 2015.

46. Kramer B, Ferrari DM, Klappa P, Pöhlmann N and Söling HD: Functional roles and efficiencies of the thioredoxin boxes of calcium-binding proteins 1 and 2 in protein folding. Biochem J 357: 83-95, 2001.

47. Zeeshan HM, Lee GH, Kim HR and Chae HJ: Endoplasmic reticulum stress and associated ROS. Int J Mol Sci 17: 327, 2016.

48. Santos CX, Nabeebaccus AA, Shah AM, Camargo LL, Filho SV and Lopes LR: Endoplasmic reticulum stress and Nox-mediated reactive oxygen species signaling in the peripheral vasculature: Potential role in hypertension. Antioxid Redox Signal 20 : $121-134,2014$

49. Kaneto H, Matsuoka T, Nakatani Y, Kawamori D, Miyatsuka T, Matsuhisa $\mathrm{M}$ and Yamasaki Y: Oxidative stress, ER stress, and the JNK pathway in type 2 diabetes. J Mol Med (Berl) 83: 429-439, 2005

50. Yuan Y, Xu X, Zhao C, Zhao M, Wang H, Zhang B, Wang N, Mao H, Zhang A and Xing C: The roles of oxidative stress, endoplasmic reticulum stress, and autophagy in aldosterone/mineralocorticoid receptor-induced podocyte injury. Lab Invest 95: 1374-1386, 2015
51. Gross ML, Hanke W, Koch A, Ziebart H, Amann KR and Ritz E: Intraperitoneal protein injection in the axolotl: The amphibian kidney as a novel model to study tubulointerstitial activation. Kidney Int 62: 51-59, 2002.

52. He F, Chen S, Wang H, Shao N, Tian X, Jiang H, Liu J, Zhu Z, Meng $X$ and Zhang C: Regulation of CD2-associated protein influences podocyte endoplasmic reticulum stress-mediated apoptosis induced by albumin overload. Gene 484: 18-25, 2011.

53. Cybulsky AV, Takano T, Papillon J, Bijian K, Guillemette J and Kennedy CR: Glomerular epithelial cell injury associated with mutant alpha-actinin-4. Am J Physiol Renal Physiol 297: F987-F995, 2009.

54. Ostergaard L, Simonsen U, Eskildsen-Helmond Y, Vorum H, Uldbjerg N, Honoré B and Mulvany MJ: Proteomics reveals lowering oxygen alters cytoskeletal and endoplasmatic stress proteins in human endothelial cells. Proteomics 9: 4457-4467, 2009.

55. Ha TS, Park HY, Seong SB and Ahn HY: Angiotensin II induces endoplasmic reticulum stress in podocyte, which would be further augmented by PI3-kinase inhibition. Clin Hypertens 21: 13-13, 2015.

56. Reiser J, Polu KR, Möller CC, Kenlan P, Altintas MM, Wei C, Faul C, Herbert S, Villegas I, Avila-Casado C, et al: TRPC6 is a glomerular slit diaphragm-associated channel required for normal renal function. Nat Genet 37: 739-744, 2005.

57. Sun X, Fang Z, Zhu Z, Yang X, He F and Zhang C: Effect of down-regulation of TRPC6 on the puromycin aminonucleoside-induced apoptosis of mouse podocytes. J Huazhong Univ Sci Technolog Med Sci 29: 417-422, 2009.

58. Chen S, He FF, Wang H, Fang Z, Shao N, Tian XJ, Liu JS, Zhu ZH, Wang YM, Wang S, et al: Calcium entry via TRPC6 mediates albumin overload-induced endoplasmic reticulum stress and apoptosis in podocytes. Cell Calcium 50: 523-529, 2011.

59. Morse E, Schroth J, You YH, Pizzo DP, Okada S, Ramachandrarao S, Vallon V, Sharma K and Cunard R: TRB3 is stimulated in diabetic kidneys, regulated by the ER stress marker CHOP, and is a suppressor of podocyte MCP-1. Am J Physiol Renal Physiol 299: F965-F972, 2010.

60. Inoki K, Mori H, Wang J, Suzuki T, Hong S, Yoshida S, Blattner SM, Ikenoue T, Rüegg MA, Hall MN, et al: mTORC1 activation in podocytes is a critical step in the development of diabetic nephropathy in mice. J Clin Invest 121: 2181-2196, 2011.

61. El Karoui K, Viau A, Dellis O, Bagattin A, Nguyen C, Baron W, Burtin M, Broueilh M, Heidet L, Mollet G, et al: Endoplasmic reticulum stress drives proteinuria-induced kidney lesions via Lipocalin 2. Nat Commun 7: 10330, 2016.

62. Nitta K, Okada K, Yanai M and Takahashi S: Aging and chronic kidney disease. Kidney Blood Press Res 38: 109-120, 2013.

63. Liu SH, Wu CT, Huang KH, Wang CC, Guan SS, Chen LP and Chiang CK: C/EBP homologous protein (CHOP) deficiency ameliorates renal fibrosis in unilateral ureteral obstructive kidney disease. Oncotarget 7: 21900-21912, 2016.

64. Kaneto H, Kajimoto Y, Miyagawa J, Matsuoka T, Fujitani Y, Umayahara Y, Hanafusa T, Matsuzawa Y, Yamasaki Y and Hori M: Beneficial effects of antioxidants in diabetes: Possible protection of pancreatic beta-cells against glucose toxicity. Diabetes 48: 2398-2406, 1999.

65. Ou Y, Hou W, Li S, Zhu X, Lin Y, Han J, Duan Z and Gui B: Sodium citrate inhibits endoplasmic reticulum stress in rats with adenine-induced chronic renal failure. Am J Nephrol 42: 14-21, 2015.

66. Kim Y, Lee H, Manson SR, Lindahl M, Evans B, Miner JH, Urano F and Chen YM: Mesencephalic astrocyte-derived neurotrophic factor as a urine biomarker for endoplasmic reticulum stress-related kidney diseases. J Am Soc Nephrol 27: 2974-2982, 2016. 